\title{
Endofibrosis in an Endurance Athlete
}

\section{Stephan Koter}

Department of Surgery, Division of Vascular Surgery, Medical University of Graz, Austria

*Corresponding author: Stephan Koter, Department of Surgery, Division of Vascular Surgery, Medical University of Graz, Austria, Tel: 43031638580670 ; E-mail: stephan.koter@medunigraz.at

Received date: 15 November 2015; Accepted date: 24 November 2015; Published date: 30 November 2015

Copyright: (c) 2015 Koter S. This is an open-access article distributed under the terms of the Creative Commons Attribution License, which permits unrestricted use, distribution, and reproduction in any medium, provided the original author and source are credited.

\section{Clinical Case Report}

A 35-year-old endurance athlete was referred for unexplained claudication-like complaints in his left lower limb. Elsewhere he was treated for functional compartment syndrome and operated for popliteal artery entrapment syndrome which proved unsuccessful. He complained of a painful and swollen left thigh at maximal exercise. Clinical investigation, ankle-to-brachial indices at rest and an additional magnetic resonance angiography were normal. An incremental treadmill test showed pathological ankle-to-brachial pressure measurement, duplex ultrasound imaging confirmed flow limitation and showed lesions of endofibrosis in the left external iliac artery. Surgery consisted of performing an endarterectomy of the external iliac artery with venous patching.

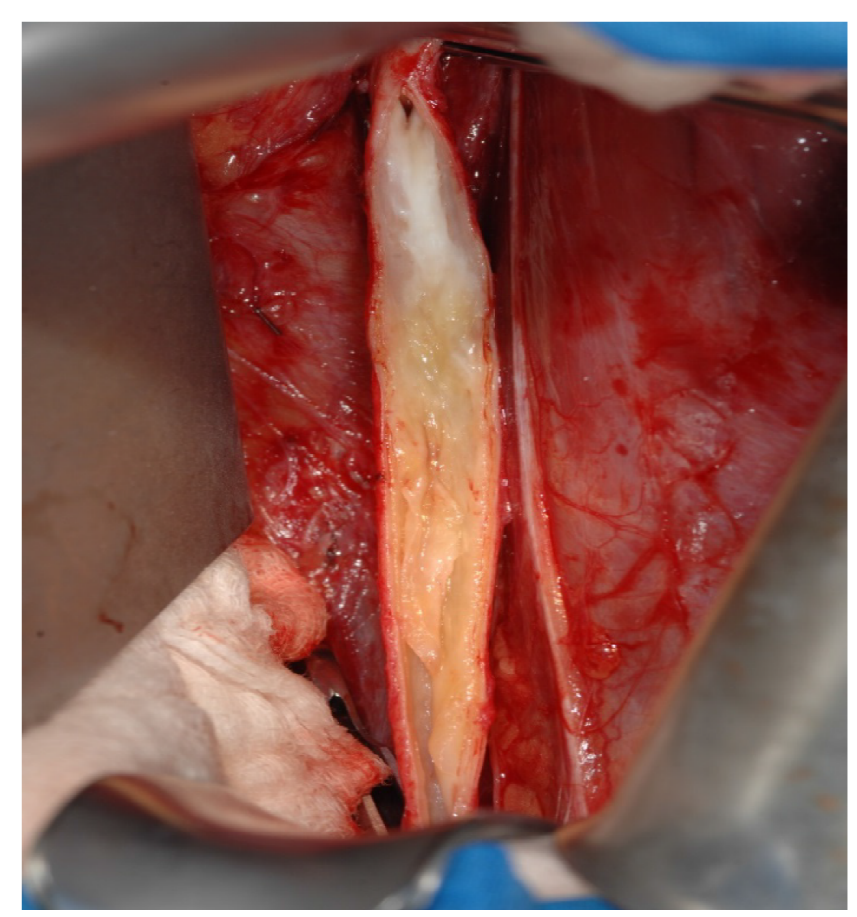

Figure 1: Endarterectomy of the external iliac artery.

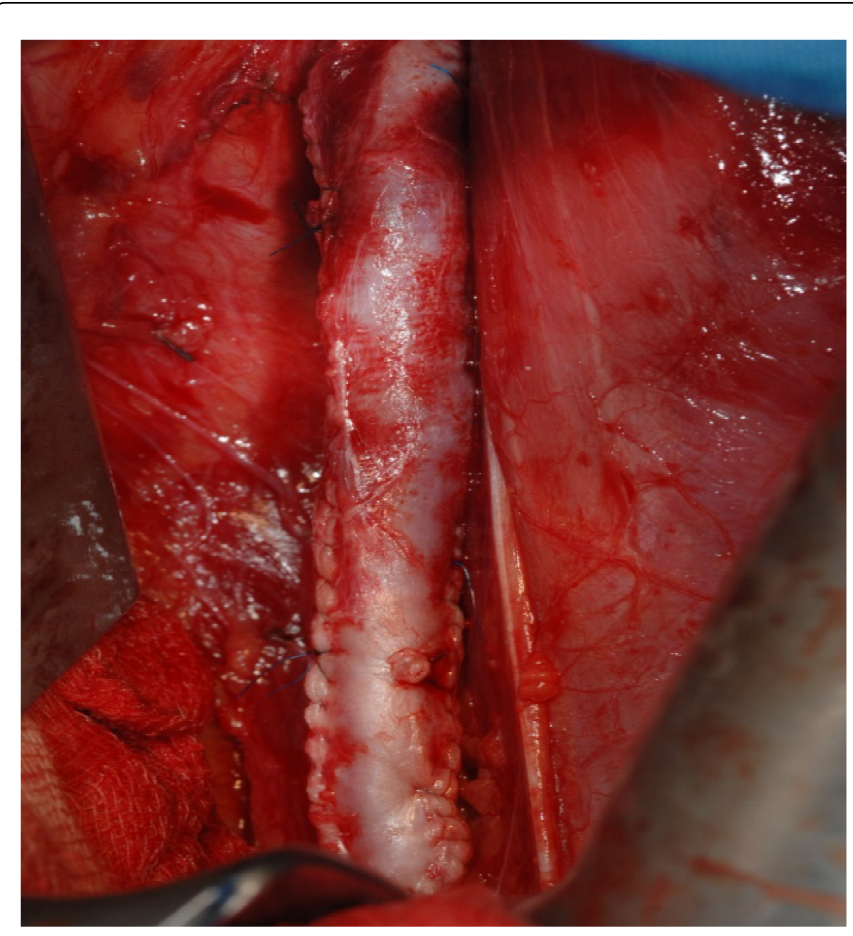

Figure 2: Surgical image of venous patching. 